\title{
Enhancement in Welding Performance of $2 \%$ Maximum Weld Repair Policy
}

\author{
Firman Edi, Suparno, M. Giatman
}

\begin{abstract}
In the implementation of welding work on a modular oil \& gas fabrication project in PT. X Batam to get welding results that are $100 \%$ very difficult to achieve and always obtain welding results through visual inspection tests and Non-Destructive Test is rejected or defect that the repair welding must be done to fix it. The causes of welding repair are classified into two, namely repair due to lack of skill welder or due to engineering aspects. With that the management of PT. $X$ Batam issued a policy of $2 \%$ maximum welding repair for each structural welding job as a KPI's and part of the company's quality manual to monitor and control of welder's performance in every project implemented. From the results of the $2 \%$ maximum KPI welding repair policy obtained significant enhancement on the performance of welders in every project undertaken and shown from the results of KPI values in 2014 the TEN FPSO E-house project was 1.2\%, in 2015 the FPSO Kraken PGM project was $1.5 \%$, in 2016 the Ghana PGM FPSO project is 0.8\%, in 2017 the Adolo Compressor FPSO project is $0.75 \%$, in 2018 the TCO Area E-house project is $0.65 \%$ and in 2019 is ongoing the BGC TEG Regeneration unit project is $1.25 \%$.
\end{abstract} policy.

Keywords : Project, welding repair, performance, KPI and

\section{INTRODUCTION}

Since 2011 the oil and gas industry has continued to develop projects that are geologically complicated, expensive, and demanding technology throughout the world. Planned new capital expenditures in key oil and gas development areas are on the rise. These projects will compete for technical expertise, essential materials, and capital, many of which will add costs and financial risks.

PT X Batam is one of the modular assemblers who participated in running and supporting the oil and gas projects throughout the world in Africa, Asia, the Middle East, Russia, Australia and America. PT X Batam are flagship 12-hectares fabrication yard is strategically located in Free Trade Zone of Batam island, Indonesia. The yard undertakes project management, detail engineering, procurement and fabrication services for major oil and gas companies. Specializing in e-houses, electric sub-stations and process equipment integrator modules, it caters for both offshore (FPSO) and onshore applications, particularly for LNG plants and headquartered office located in Malaysia.

In the modularization construction works that there are several work breakdown structures (WBS) are structural, architectural, piping, painting, mechanical, electrical, instrument, F\&G, HVAC and telecommunication. Where the biggest contribute in WBS is welding work. In the implementation of welding work on a modular oil \& gas fabrication project at PT. X Batam, to get welding results that are $100 \%$ very difficult to achieve and always obtain welding results through visual inspection tests and Non-Destructive Test is rejected or defect that the repair welding must be done to fix it.

The causes of welding repair are classified into two, namely repair due to lack of skill welder or due to engineering aspects. Welding defects that are commonly found such as porosity, slag inclusion, lack of fusion, undercut generally come from inadequate welder skills to conduct good welding which have an impact on overhead costs, quality and time of the project according to Firman Edi researched in 2017[1]. While welding defects due to engineering aspects can be in the form of defects such as crack and distortion which cause changes in the mechanical properties of the material in the HAZ area and weld ability of the base metal itself according to Gani Trisdyanto and Mochamad Choifin researched in 2017[2] .

With that the management of PT. X Batam issued a policy of $2 \%$ maximum welding repair for each structural welding job as a KPI's and part of the company's quality manual to monitor and control of welders performance in every project implemented.

\section{MATERIALS AND METHODS}

Policy is a provision that shows the direction and guidelines for action. Policies are generally made by institutional leaders, both government and private institutions. One good policy indicator is made scientifically, that is, a policy is made rationally and based on complete, accurate and up-to-date data according to Sugiyono,2017[3].

This policy research uses a policy evaluation research method approach issued by the management of PT X Batam to get the relationship between program policies and the results of the performance welding work on each project implemented.

Revised Manuscript Received on January 10, 2020.

* Correspondence Author

Firman Edi*, Technology \& Vocational Eduction Program, Padang State University, Padang, Indonesia. Email: firmanedi972000@yahoo.com

Suparno, Technology \& Vocational Eduction Program, Padang State University, Padang, Indonesia.

M. Giatman, Technology \& Vocational Eduction Program, Padang State University, Padang, Indonesia.

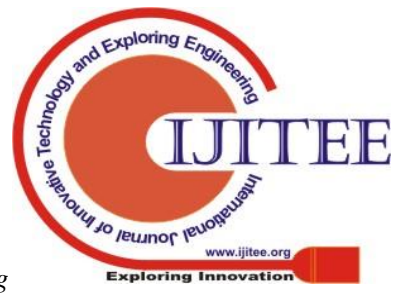


Enhancement in Welding Performance of 2\% Maximum Weld Repair Policy

A. Weld Repair Policy (KPI)

Table-I. Weld Repair Policy

\begin{tabular}{|c|c|c|c|}
\hline KPI & Metric Name & Metric Definition \& Measure & Target \\
\hline \multirow{3}{*}{ QAQC } & \multirow{3}{*}{ Weld Repair } & $\begin{array}{l}\text { WELD REPAIRS (STRUCTURAL) } \\
\text { The Percentage To Date Of: (Welds Performed By Wasco For } \\
\text { Steel Plate/ Steel Structures That Needed Repairs)/ (The Total } \\
\text { Number Of Such Weld) }\end{array}$ & $<2 \%$ \\
\hline & & $\begin{array}{l}\text { WELD REPAIRS (PIPING) } \\
\text { The Percentage To Date Of: (Weld length Performed By Wasco } \\
\text { For Piping That Needed Repairs) / (The Total Length Of Such } \\
\text { Welds) }\end{array}$ & $<2 \%$ \\
\hline & & $\begin{array}{l}\text { WELD REPAIRS (PROCESS EQUIPMENT) } \\
\text { The Percentage To Date Of: (Welds Performed By Wasco } \\
\text { During And Process Equipment Welds That Need To Be } \\
\text { Repaired (By Joint) / The Total Number Of Such Weld. }\end{array}$ & $<2 \%$ \\
\hline
\end{tabular}

B. Welding Processes Flowchart
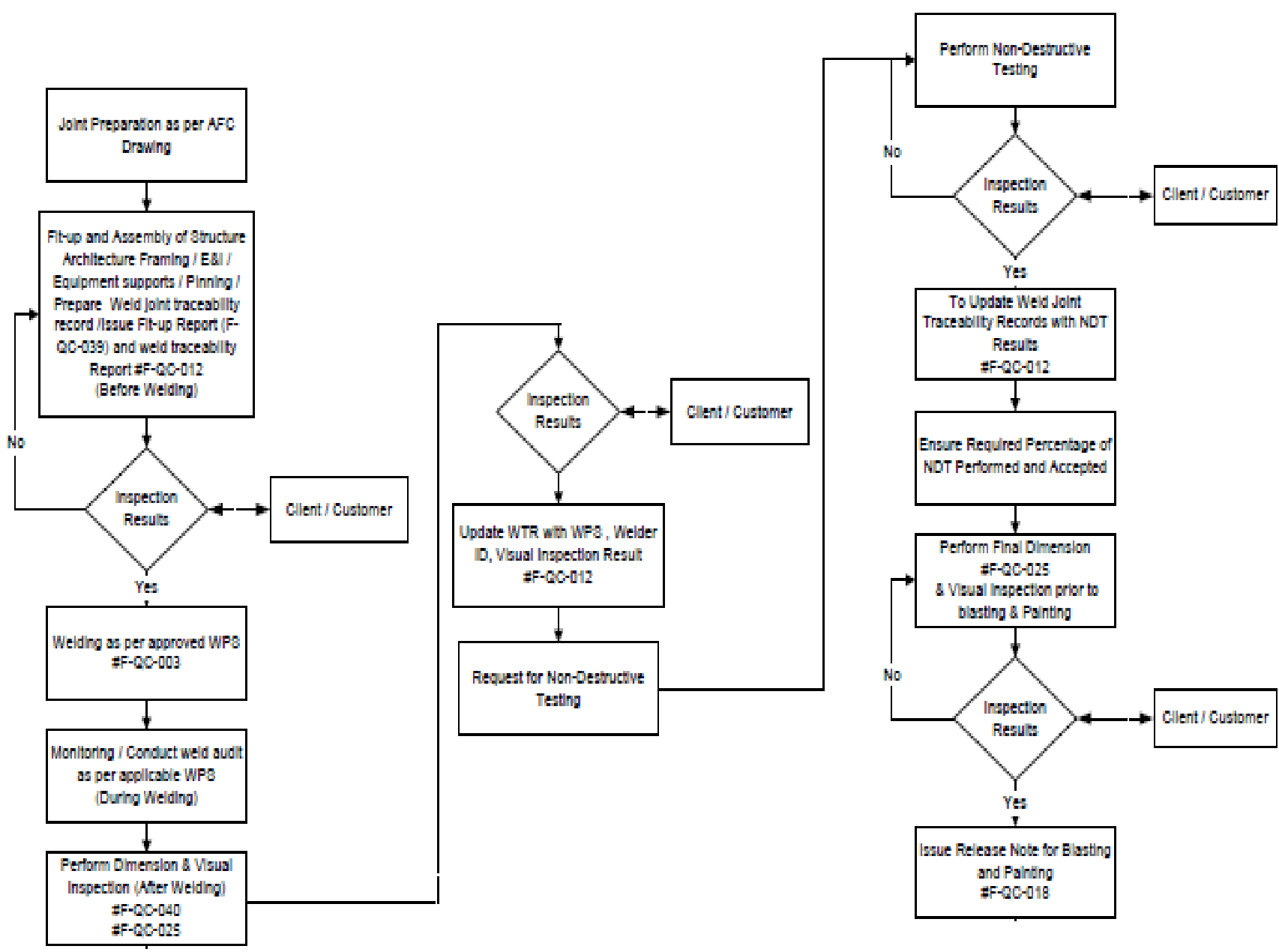

Figure 1. Welding Process Flowchart at PT X Batam 


\section{RESULTS AND DISCUSSION}

\section{A. Result Based on Project Wise}

\section{Table-II TEN FPSO Project KPI in 2014}

\begin{tabular}{|c|c|c|c|c|c|c|c|c|}
\hline \multicolumn{2}{|c|}{\begin{tabular}{|l|l|} 
\\
\end{tabular}} & \multicolumn{7}{|c|}{ QA - PROJECT KEY PERFORMANCE INDICATOR } \\
\hline \multirow{2}{*}{\multicolumn{2}{|c|}{$\frac{\text { P ROJECT TITLE }}{\text { MODULE }}$}} & \multicolumn{7}{|c|}{ TEN FPSO EHOUSE PROJECT } \\
\hline & & \multicolumn{7}{|l|}{ M-09 } \\
\hline & \multicolumn{7}{|l|}{ WEF-176 } \\
\hline \multicolumn{2}{|c|}{\begin{tabular}{|l} 
P ROJECT NO \\
CUT OF DATE
\end{tabular}} & $2013-12-01-2014-12-$ & & & & & & \\
\hline \multicolumn{9}{|c|}{ 1.0 P ROJECT WELD REP AIR DATE } \\
\hline & & \multicolumn{4}{|c|}{ ACCUMULATIVE (TOTAL) } & \multicolumn{3}{|l|}{ WEEKLY ACHIEVEMENT } \\
\hline & & \multicolumn{2}{|c|}{ LENGTH TES TED R T or UT } & \multicolumn{2}{|c|}{ LENGTH REP AIR RT or UT } & LENGTH TESTED R T or UT & \multicolumn{2}{|c|}{ LENGTH REP AIR RT or UT } \\
\hline \multicolumn{2}{|r|}{ DIS C IP LINE / SCOP E OF WOR K } & \multicolumn{2}{|l|}{$(\mathrm{mm}) / \mathrm{J}$ OINT } & $(\mathrm{mm}) / \mathrm{J}$ OINT & $\%$ & $(\mathrm{~mm}) / \mathrm{J}$ OINT & $(\mathrm{mm}) / \mathrm{J} \mathrm{OINT}$ & $\%$ \\
\hline Stru & cture & 1,060 & & 12730 & $1.20 \%$ & $1,060,854$ & 12730 & $1.20 \%$ \\
\hline \multicolumn{9}{|c|}{ 2.0 P ROJECT WELDERS P ERFORMANCE RECORDS } \\
\hline \multirow{2}{*}{\multicolumn{2}{|c|}{ WELDER/WELDING OP ERATOR }} & \multicolumn{3}{|l|}{\begin{tabular}{|l} 
ACCUMULATIVE (TOTAL) \\
\end{tabular}} & \multicolumn{4}{|c|}{ WEEKLY ACHEVEMENT } \\
\hline & & LENGTH TES TED R T or UT & \multicolumn{2}{|c|}{ LENGTH REP AIR RT or UT } & \multicolumn{2}{|c|}{ LENGTH TESTED RT or UT } & \multicolumn{2}{|c|}{ LENGTH REP AIR RT or UT } \\
\hline NO & NAME & $(\mathrm{mm})$ & $(\mathrm{mm})$ & $\%$ & & $(\mathrm{~mm})$ & $(\mathrm{mm})$ & $\%$ \\
\hline 1 & B. 1028 & 360.00 & 0.00 & $0.00 \%$ & & 360.00 & 0.00 & $0.00 \%$ \\
\hline 2 & B. 1040 & $5,781.00$ & 0.00 & $0.00 \%$ & & $5,781.00$ & 0.00 & $0.00 \%$ \\
\hline 3 & B. 1051 & 455.00 & 0.00 & $0.00 \%$ & & 455.00 & 0.00 & $0.00 \%$ \\
\hline 4 & B. 1053 & $3,596.00$ & 150.00 & $4.17 \%$ & & $3,596.00$ & 150.00 & $4.17 \%$ \\
\hline 10 & B. 1140 & $46,652.00$ & 0.00 & $0.00 \%$ & & $46,652.00$ & 0.00 & $0.00 \%$ \\
\hline 11 & B.1144 & 482.00 & 0.00 & $0.00 \%$ & & 482.00 & 0.00 & $0.00 \%$ \\
\hline 34 & B.1916 & $34,581.00$ & 100.00 & $0.29 \%$ & & $34,581.00$ & 100.00 & $0.29 \%$ \\
\hline 35 & B.1918 & $29,463.00$ & 40.00 & $0.14 \%$ & & $29,463.00$ & 40.00 & $0.14 \%$ \\
\hline 36 & B.1920 & $11,108.00$ & 0.00 & $0.00 \%$ & & $11,108.00$ & 0.00 & $0.00 \%$ \\
\hline \begin{tabular}{|l|}
37 \\
\end{tabular} & B.1921 & $35,689.00$ & 0.00 & $0.00 \%$ & & $35,689.00$ & 0.00 & $0.00 \%$ \\
\hline 38 & B. 1925 & $13,576.00$ & 0.00 & $0.00 \%$ & & $13,576.00$ & 0.00 & $0.00 \%$ \\
\hline
\end{tabular}

Table-III KRAKEN FPSO Project KPI in 2015

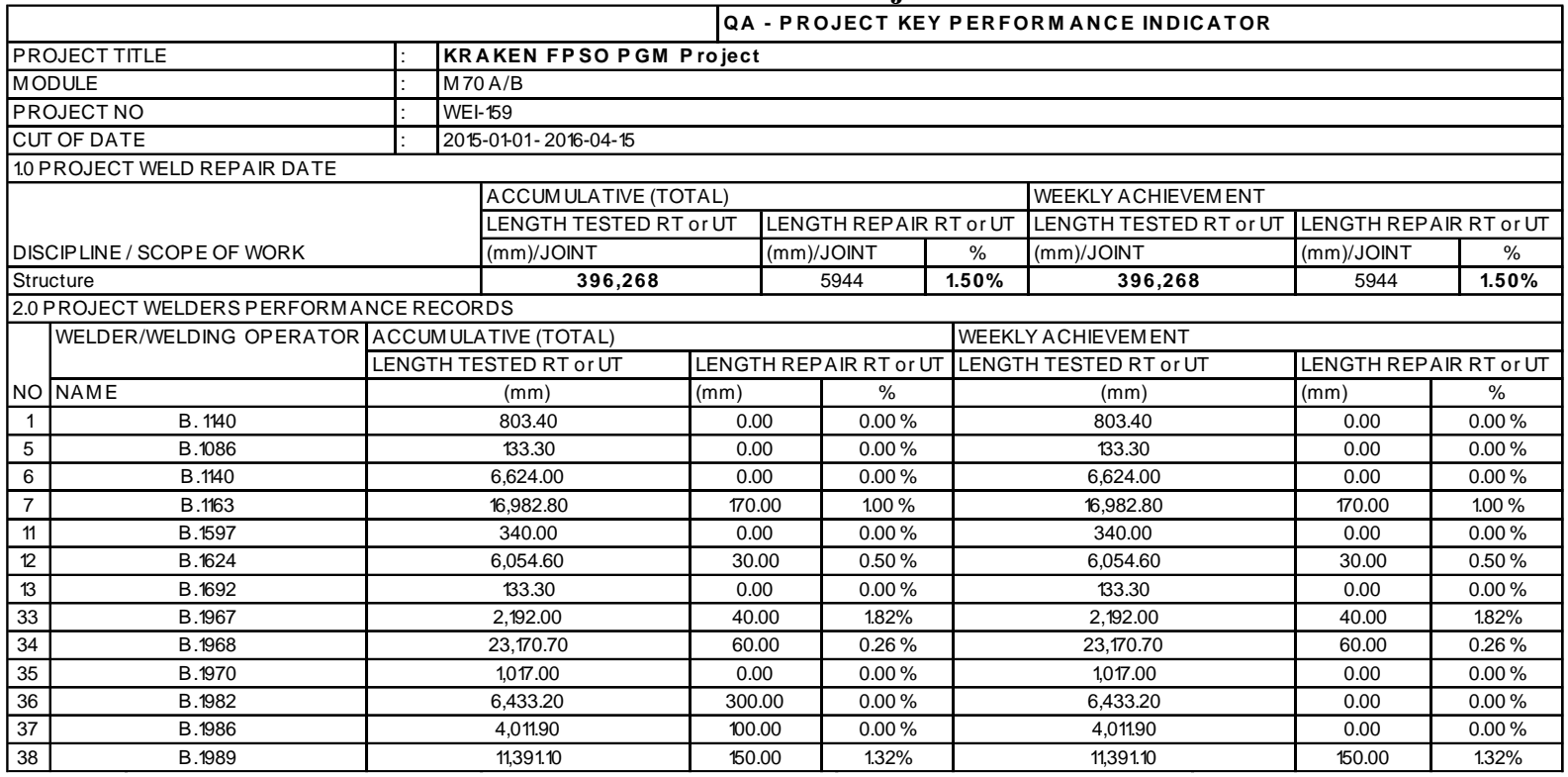

Table-IV YINSON GHANA FPSO Project KPI in 2016

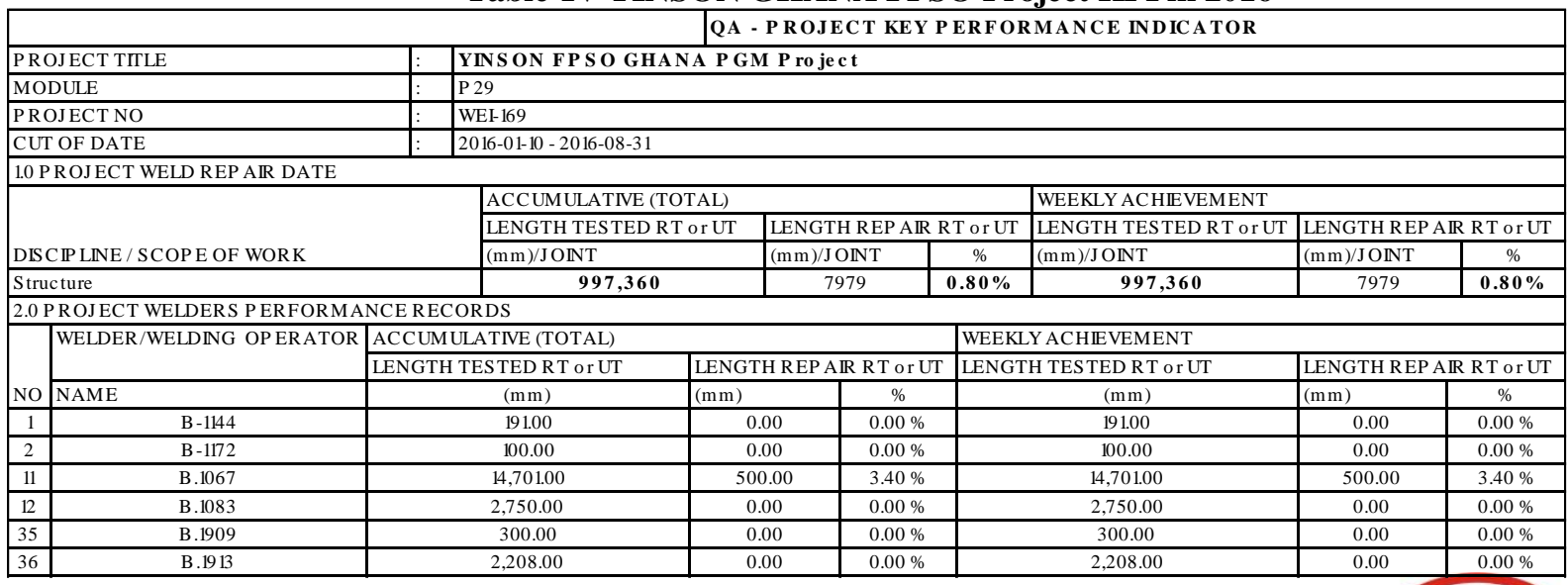


Enhancement in Welding Performance of 2\% Maximum Weld Repair Policy

Table-V BW ADOLO Compressor Project KPI in 2017

\begin{tabular}{|c|c|c|c|c|c|c|c|c|c|}
\hline \multicolumn{10}{|c|}{ QA - PROJECT KEY PERFORMANCE INDICATOR } \\
\hline \multicolumn{2}{|c|}{ PROJECT TITLE } & $:$ & \multicolumn{7}{|c|}{4396 - Azurite Redeployment for Ruche-Gas Lift Compressor } \\
\hline \multicolumn{2}{|c|}{ MODULE } & : & \multicolumn{7}{|l|}{ M23 } \\
\hline \multicolumn{2}{|c|}{ PROJECT NO } & : & \multicolumn{7}{|l|}{ CP 00017} \\
\hline \multicolumn{2}{|c|}{ CUT OF DATE } & : & \multicolumn{7}{|c|}{$2017-05-01-2018-05-28$} \\
\hline \multicolumn{10}{|c|}{ 1.0 P ROJECT WELD REP AIR DATE } \\
\hline \multirow{3}{*}{\multicolumn{2}{|c|}{ DIS C IP LINE / S COP E OF WOR K }} & & \multicolumn{4}{|c|}{ ACCUMULATIVE (TOTAL) } & \multicolumn{3}{|c|}{ WEEKLY ACHIEVEMENT } \\
\hline & & & \multirow{2}{*}{\begin{tabular}{|l|} 
LENGTH TES TED R T or UT \\
$(\mathrm{mm}) / \mathrm{J}$ ONT
\end{tabular}} & \begin{tabular}{l|l} 
or UT & LENG
\end{tabular} & \multicolumn{2}{|c|}{ LENGTH REP AIR RT or UT } & LENGTH TESTED R T or UT & \multicolumn{2}{|c|}{ LENGTH REP AIR RT or UT } \\
\hline & & & & $(\mathrm{mm}) /$ & INT & $\%$ & $(\mathrm{~mm}) / \mathrm{JONT}$ & $(\mathrm{mm}) / \mathrm{JOIN}$ & $\%$ \\
\hline & & & 366 , & & 46 & $0.75 \%$ & 366,085 & 2746 & $0.75 \%$ \\
\hline \multicolumn{10}{|c|}{$\begin{array}{l}\text { 2.0 P ROJECT WELDERS P ERFOR MANCE RECOR DS } \\
\end{array}$} \\
\hline \multirow{2}{*}{\multicolumn{2}{|c|}{ WELDER/WELDING OP ERATOR }} & \multicolumn{4}{|c|}{ ACCUMULATIVE (TOTAL) } & \multicolumn{4}{|c|}{ WEEKLY ACHEVEMENT } \\
\hline & & \multicolumn{2}{|c|}{ LENGTH TESTED RT or UT } & \multicolumn{2}{|c|}{ LENGTH REP AIR R T or UT } & \multicolumn{2}{|c|}{ LENGTH TESTED RT or UT } & \multicolumn{2}{|c|}{ LENGTH REP AIR RT or UT } \\
\hline NO & NAME & & $(\mathrm{mm})$ & $(\mathrm{mm})$ & $\%$ & & $(\mathrm{~mm})$ & $(\mathrm{mm})$ & $\%$ \\
\hline 1 & B-1083 & & 528.00 & 0.00 & $0.00 \%$ & & 528.00 & 0.00 & $0.00 \%$ \\
\hline 2 & B.1069 & & 268.00 & 0.00 & $0.00 \%$ & & 268.00 & 0.00 & $0.00 \%$ \\
\hline \begin{tabular}{|l|}
3 \\
\end{tabular} & B. 1083 & & $26,184.00$ & 150.00 & $0.57 \%$ & & $26,184.00$ & 150.00 & $0.57 \%$ \\
\hline 4 & B.1086 & & $17,603.00$ & 0.00 & $0.00 \%$ & & $17,603.00$ & 0.00 & $0.00 \%$ \\
\hline 5 & B. 1163 & & $4,892.00$ & 0.00 & $0.00 \%$ & & $4,892.00$ & 0.00 & $0.00 \%$ \\
\hline 6 & B.1172 & & $35,505.00$ & 150.00 & $0.42 \%$ & & $35,505.00$ & 150.00 & $0.42 \%$ \\
\hline \begin{tabular}{|l|l|}
7 \\
\end{tabular} & B.1196 & & $10,430.00$ & 0.00 & $0.00 \%$ & & $10,430.00$ & 0.00 & $0.00 \%$ \\
\hline 8 & B.1242 & & 300.00 & 0.00 & $0.00 \%$ & & 300.00 & 0.00 & $0.00 \%$ \\
\hline \begin{tabular}{|c|}
9 \\
\end{tabular} & B.1247 & & $32,320.00$ & 769.00 & $2.38 \%$ & & $32,320.00$ & 769.00 & $2.38 \%$ \\
\hline 10 & B. 1313 & & 770.00 & 0.00 & $0.00 \%$ & & 770.00 & 0.00 & $0.00 \%$ \\
\hline 32 & B. 1952 & & $3,329.00$ & 460.00 & $13.82 \%$ & & $3,329.00$ & 460.00 & $13.82 \%$ \\
\hline \begin{tabular}{|l|}
33 \\
\end{tabular} & B.1956 & & $\begin{array}{l}2,451.00 \\
\end{array}$ & 0.00 & $0.00 \%$ & & $12,451.00$ & 0.00 & $0.00 \%$ \\
\hline 34 & B. 1958 & & $10,394.00$ & 440.00 & $4.23 \%$ & & $10,394.00$ & 440.00 & $4.23 \%$ \\
\hline 35 & B.1959 & & $3,256.00$ & 0.00 & $0.00 \%$ & & $3,256.00$ & 0.00 & $0.00 \%$ \\
\hline \begin{tabular}{|l|}
36 \\
\end{tabular} & B.1960 & & $8,916.00$ & 595.00 & $6.67 \%$ & & $8,916.00$ & 595.00 & $6.67 \%$ \\
\hline 37 & B.1969 & & $1,324.00$ & 0.00 & $0.00 \%$ & & $1,324.00$ & 0.00 & $0.00 \%$ \\
\hline 38 & B.1972 & & $1,116.00$ & 0.00 & $0.00 \%$ & & $1,116.00$ & 0.00 & $0.00 \%$ \\
\hline
\end{tabular}

Table-VI TCO Future Growth Project KPI in 2018

\begin{tabular}{|c|c|c|c|c|c|c|c|c|c|}
\hline \multirow{2}{*}{\multicolumn{3}{|c|}{\begin{tabular}{|l} 
\\
P ROJECT TITLE
\end{tabular}}} & & \multicolumn{6}{|c|}{ QA - PROJECT KEY PERFORMANCE INDICATOR } \\
\hline & & & \multicolumn{7}{|c|}{ FUTURE GROWTH PROJECT - GATHERING } \\
\hline \multicolumn{3}{|c|}{ MODULE } & \multicolumn{7}{|l|}{ 51-SU-3301 } \\
\hline \multicolumn{3}{|c|}{ PROJECT NO } & \multicolumn{7}{|l|}{ EH-00011-001 } \\
\hline \multicolumn{3}{|c|}{ CUT OF DATE } & \multicolumn{7}{|c|}{$2018-02-01-2018-06-28$} \\
\hline \multicolumn{10}{|c|}{ 1.0 P ROJECT WELD REP AIR DATE } \\
\hline \multirow{3}{*}{\multicolumn{3}{|c|}{ DIS C IP LINE / SCOP E OF WORK }} & \multicolumn{4}{|c|}{ ACCUMULATIVE (TOTAL) } & \multicolumn{3}{|c|}{ WEEKLY ACHEVEMENT } \\
\hline & & & \multicolumn{2}{|c|}{ LENGTH TESTED RT or UT } & \multicolumn{2}{|c|}{ LENGTH REP AIR RT or UT } & LENGTH TES TED RT or UT & \multicolumn{2}{|c|}{ LENGTH REP AIR RT or UT } \\
\hline & & & & $(\mathrm{mm}) / \mathrm{J}$ OINT & $\%$ & $(\mathrm{~mm}) / \mathrm{JONT}$ & $(\mathrm{mm}) / \mathrm{J}$ OINT & $\%$ \\
\hline \multicolumn{3}{|c|}{ Structure } & \multirow{2}{*}{\multicolumn{2}{|c|}{157,701}} & 1025.1 & $0.65 \%$ & 157,701 & 1025.1 & $0.65 \%$ \\
\hline \multicolumn{9}{|c|}{ 2.0 P ROJECT WELDERS P ERFORMANCE RECORDS } & \\
\hline \multirow{2}{*}{\multicolumn{2}{|c|}{ WELDER/WELDING OP ER ATOR }} & \multicolumn{4}{|c|}{ ACCUMULATIVE (TOTAL) } & \multicolumn{4}{|c|}{ WEEKLY ACHEVEMENT } \\
\hline & & \multicolumn{2}{|c|}{ LENGTH TES TED RT or UT } & LENGTH RE & RT or UT & LENGTI & H TES TED RT or UT & LENGTH RE & RT or UT \\
\hline NO & NAME & & $(\mathrm{mm})$ & $(\mathrm{mm})$ & $\%$ & & $(\mathrm{~mm})$ & $(\mathrm{mm})$ & $\%$ \\
\hline 1 & B.1196 & & $1,799.30$ & 0.00 & $0.00 \%$ & & $1,799.30$ & 0.00 & $0.00 \%$ \\
\hline 2 & B. 1242 & & $1,691.20$ & 0.00 & $0.00 \%$ & & $1,691.20$ & 0.00 & $0.00 \%$ \\
\hline 3 & B. 1389 & & $1,091.20$ & 0.00 & $0.00 \%$ & & $1,091.20$ & 0.00 & $0.00 \%$ \\
\hline 4 & B. 1561 & & $2,523.20$ & 0.00 & $0.00 \%$ & & $2,523.20$ & 0.00 & $0.00 \%$ \\
\hline 5 & B. 1624 & & $1,424.60$ & 0.00 & $0.00 \%$ & & $1,424.60$ & 0.00 & $0.00 \%$ \\
\hline 6 & B. 1703 & & $25,401.00$ & 533.20 & $2.10 \%$ & & $25,401.00$ & 533.20 & $2.10 \%$ \\
\hline 7 & B. 1903 & & 666.40 & 0.00 & $0.00 \%$ & & 666.40 & 0.00 & $0.00 \%$ \\
\hline 8 & B. 1925 & & $7,160.00$ & 0.00 & $0.00 \%$ & & $7,160.00$ & 0.00 & $0.00 \%$ \\
\hline \begin{tabular}{|l|}
9 \\
\end{tabular} & B. 1929 & & $23,573.60$ & 0.00 & $0.00 \%$ & & $23,573.60$ & 0.00 & $0.00 \%$ \\
\hline 10 & B. 1941 & & $7,997.90$ & $1,280.00$ & $16.00 \%$ & & $7,997.90$ & $1,280.00$ & $16.00 \%$ \\
\hline \begin{tabular}{|l|}
11 \\
\end{tabular} & B.1944 & & $7,231.40$ & 0.00 & $0.00 \%$ & & $7,231.40$ & 0.00 & $0.00 \%$ \\
\hline 12 & B. 1948 & & $9,286.00$ & 0.00 & $0.00 \%$ & & $9,286.00$ & 0.00 & $0.00 \%$ \\
\hline 13 & B. 1950 & & $4,363.80$ & 0.00 & $0.00 \%$ & & $4,363.80$ & 0.00 & $0.00 \%$ \\
\hline 14 & B. 1970 & & $6,251.50$ & 0.00 & $0.00 \%$ & & $6,251.50$ & 0.00 & $0.00 \%$ \\
\hline 15 & B. 2030 & & 800.00 & 0.00 & $0.00 \%$ & & 800.00 & 0.00 & $0.00 \%$ \\
\hline 16 & B.2033 & & $15,547.70$ & 266.60 & $1.71 \%$ & & $15,547.70$ & 266.60 & $1.71 \%$ \\
\hline 17 & B.2033/1703 & & 800.00 & 0.00 & $0.00 \%$ & & 800.00 & 0.00 & $0.00 \%$ \\
\hline 18 & B.467 & & $18,360.50$ & 60.00 & $0.33 \%$ & & $18,360.50$ & 60.00 & $0.33 \%$ \\
\hline \begin{tabular}{|l|l|}
19 \\
\end{tabular} & B. 903 & & $6,546.00$ & 90.00 & $1.37 \%$ & & $6,546.00$ & 90.00 & $1.37 \%$ \\
\hline \begin{tabular}{|l|l}
20 \\
\end{tabular} & B.910 & & $9,968.00$ & 0.00 & $0.00 \%$ & & $9,968.00$ & 0.00 & $0.00 \%$ \\
\hline \begin{tabular}{|l|l|}
21 \\
\end{tabular} & B. $910 / B .497$ & & $3,200.00$ & 0.00 & $0.00 \%$ & & $3,200.00$ & 0.00 & $0.00 \%$ \\
\hline \begin{tabular}{|l|l|}
22 \\
\end{tabular} & B. 934 & & $1,218.00$ & 0.00 & $0.00 \%$ & & $1,218.00$ & 0.00 & $0.00 \%$ \\
\hline 23 & В 1929 & & 800.00 & 0.00 & $0.00 \%$ & & 800.00 & 0.00 & $0.00 \%$ \\
\hline
\end{tabular}


Table-VII BGC TEG Project KPI in 2019 (Ongoing Construction)

\begin{tabular}{|c|c|c|c|c|c|c|c|c|c|}
\hline \multirow{2}{*}{\multicolumn{3}{|c|}{\begin{tabular}{|l|} 
P ROJECT TITLE \\
\end{tabular}}} & & \multicolumn{6}{|c|}{ QA - PROJECT KEY PERFORMANCE INDICATOR } \\
\hline & & & \multicolumn{7}{|c|}{ Gas Dehydration \& TEG Regeneration Sys tem } \\
\hline \multicolumn{3}{|c|}{ MODULE } & \multicolumn{7}{|l|}{$\mathrm{C} 027$} \\
\hline \multicolumn{3}{|c|}{ PROJECT NO } & \multicolumn{7}{|l|}{ WEICP -021 } \\
\hline \multicolumn{3}{|c|}{ CUT OF DATE } & \multicolumn{7}{|c|}{$2019-09-18-2019-11-15$} \\
\hline \multicolumn{10}{|c|}{ 1.0 P ROJECT WELD REP AIR DATE } \\
\hline \multirow{3}{*}{\multicolumn{3}{|c|}{ DIS C IP LINE / S COP E OF WOR K }} & \multicolumn{4}{|c|}{ ACCUMULATIVE (TOTAL) } & \multicolumn{3}{|c|}{ WEEKLY ACHEVEMENT } \\
\hline & & & \multicolumn{2}{|c|}{ LENGTH TESTED RT or UT } & \multicolumn{2}{|c|}{ LENGTH REP AIR RT or UT } & LENGTH TESTED RT or UT & \multicolumn{2}{|c|}{ LENGTH REP AIR RT or UT } \\
\hline & & & \multicolumn{2}{|c|}{$(\mathrm{mm}) / \mathrm{J}$ OINT } & $(\mathrm{mm}) / \mathrm{J}$ OINT & $\%$ & $(\mathrm{~mm}) / \mathrm{J}$ ONT & $(\mathrm{mm}) / \mathrm{JONT}$ & $\%$ \\
\hline \multicolumn{3}{|c|}{ Structure } & \multirow{2}{*}{\multicolumn{2}{|c|}{91,420}} & 1142.8 & $1.25 \%$ & 157,701 & 1142.8 & $1.25 \%$ \\
\hline \multicolumn{9}{|c|}{ 2.0 P ROJECT WELDERS P ERFORMANCE RECORDS } & \\
\hline & \multirow{2}{*}{ WELDER/WELDING OP ER ATOR } & \multicolumn{4}{|c|}{ ACCUMULATIVE (TOTAL) } & \multicolumn{4}{|c|}{ WEEKLY ACHEVEMENT } \\
\hline & & \multicolumn{2}{|c|}{ LENGTH TESTED R T or UT } & \multicolumn{2}{|c|}{ LENGTH REP AIR RT or UT } & \multicolumn{2}{|c|}{ LENGTH TESTED RT or UT } & \multicolumn{2}{|c|}{ LENGTH REP AIR RT or UT } \\
\hline $\mathrm{NO}$ & NAME & & $(\mathrm{mm})$ & $(\mathrm{mm})$ & $\%$ & & $(\mathrm{~mm})$ & $(\mathrm{mm})$ & $\%$ \\
\hline 1 & B.1163 & & $4,425.00$ & 50.00 & $1.13 \%$ & & $4,425.00$ & 50.00 & $1.13 \%$ \\
\hline 2 & B. 1242 & & $4,359.00$ & 50.00 & $1.15 \%$ & & $4,359.00$ & 50.00 & $1.15 \%$ \\
\hline 3 & B. 1507 & & $7,868.00$ & 50.00 & $0.64 \%$ & & $7,868.00$ & 50.00 & $0.64 \%$ \\
\hline 4 & B.1561 & & $7,150.00$ & 40.00 & $0.56 \%$ & & $7,150.00$ & 40.00 & $0.56 \%$ \\
\hline 5 & B.1597 & & $9,422.00$ & 0.00 & $0.00 \%$ & & $9,422.00$ & 0.00 & $0.00 \%$ \\
\hline 6 & B. 1703 & & $6,480.00$ & 130.00 & $2.01 \%$ & & $6,480.00$ & 130.00 & $2.01 \%$ \\
\hline \begin{tabular}{|l|}
7 \\
\end{tabular} & B.1915 & & $1,560.00$ & 0.00 & $0.00 \%$ & & $1,560.00$ & 0.00 & $0.00 \%$ \\
\hline 8 & B.1929 & & $4,091.00$ & 105.00 & $2.57 \%$ & & $4,091.00$ & 105.00 & $2.57 \%$ \\
\hline \begin{tabular}{|l|}
9 \\
\end{tabular} & B.1936 & & $7,166.00$ & 90.00 & $1.26 \%$ & & $7,166.00$ & 90.00 & $1.26 \%$ \\
\hline
\end{tabular}

\section{B. Summary of KPI's Achievement}

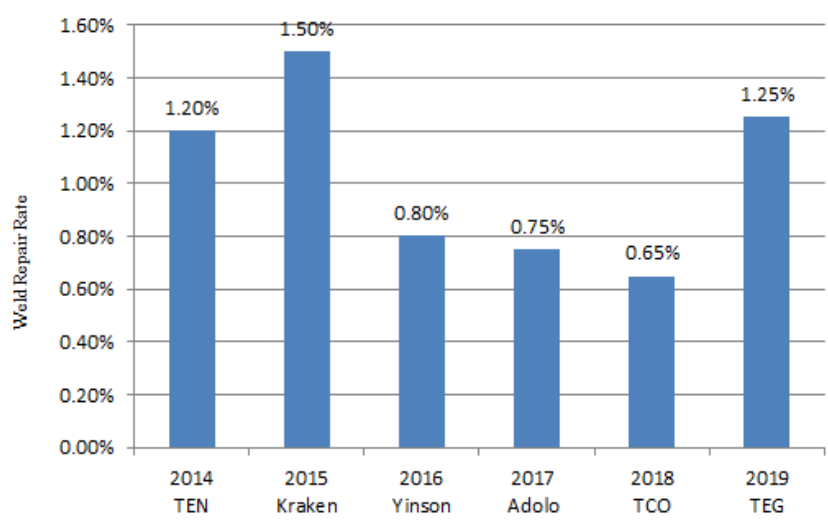

Fig. 2. Summary KPI's Achievement of Repair Rate < $\mathbf{2 . 0 \%}$

\section{Discussion}

As an evaluation of the maximum welding repair policy (KPI) of $2 \%$, there was a significant increase in the performance of welders in each project carried out and shows the KPI value in figure 2 with the results in 2014 the TEN FPSO Ehouse project was $1.2 \%$, in 2015 the PGM FPSO project Kraken is $1.5 \%$, in 2016 the PGM Ghana FPSO project is $0.8 \%$, in 2017 the Adolo BW Compressor FPSO project is $0.75 \%$, in 2018 the TCO Area Ehouse project is $0.65 \%$ and in 2019 Ongoing Regeneration of TEG BGC project units is $1.25 \%$.

\section{CONCLUSION}

Based on the above statement, it can be concluded that the implementation of welding repair policies (KPI) can influence and improve the quality performance of welders and as a reference to be measured and controlled in the implementation of welding work in each project. Firman Edi, as student at Technology \& Vocational Eduction Program, Padang State University, Padang, Indonesia. Email: firmanedi972000@yahoo.com

\section{ACKNOWLEDGMENT}

This research is very useful and supportive at PT X, where researchers have also been project managers since 2012 in developing a quality management system.

\section{REFERENCES}

1. Firman Edi, (2017). Implementation of FMEA and FTA Method for Predicting Delivery Time Project EPC - Case Study of Power Generation Modules 2x62MW Fabrication FPSO X Project PT. X in Batam, Jurnal Ilmiah TEKNOBIZ, 07:3, 129-136.

2. Gani Trisdyanto, Mochamad Choifin, (2019). Analysis of the Effects of Multiple Repair and Non-Repair Variations on FCAW Welding of JIS G3106 SM 490 YB Carbon Steel Against Mechanical Properties, G-Tech Jurnal Teknology Terapan F.SAINTEK Unira Malang, 2:2, 53-61.

3. Prof Dr. Sugiyono, (2017). Policy Research Methods, iii, ISBN No.978-602-289-313, ALFABETA, Bandung.

4. Yousaf, F., Ikramullah Butt, S., (2014). Reduction in Repair rate of Welding Processes by Determination \& Controlling of Critical KPIVs, International Journal of Production Management and Engineering, 2(1), 23-36.

5. Flaig, J.J. (2009). A unifying process capability metric. Journal of Industrial Engineering and Management, 2(1), 48-59.

6. ASME IX. (2010). Newyork: Qualification Standard for Welding and Brazing procedure, welder, brazer and Welding and Brazing Operator

7. AWS. (2015). AWS D1.1/D1.1M Structural Welding Code - Steel Miami: American Welding Society.

8. Cecep Slamet Abadi, Rosidi, Idrus Assagaf, (2019). Analisa Kekuatan Welding Repair Baja AISI 420 Dengan Metoda GMAW, Journal Politeknologi, 18:3, 297-306.

9. Aftoni Heri Septian Pamungkas, Budi Harjanto, Indah Widiastuti, (2019). Quality Analysis of Repairing Cast Aluminum Wheel Repair Using the Oxy-Acetyline Welding Method with Preheating and PWHT Treatment, Nozel Jurnal Pendidikan Teknik Mesin, 01:01, 23-28.

10. Yoshihiro Yamashita, (2001). Newly Undertaken Inspection and Repairs for Aged Nuclear Power Generators, Journal of Nuclear Science and Technology, 38:10, 887-890.

\section{AUTHORS PROFILE}

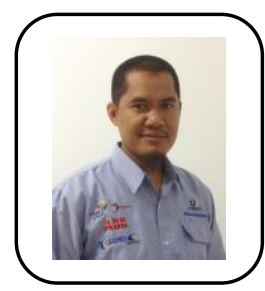

Firman Edi, as $\mathrm{PhD}$ student at Technology \& Vocational Eduction Program, Padang State University, Padang, Indonesia. Email: firmanedi972000@yahoo.com

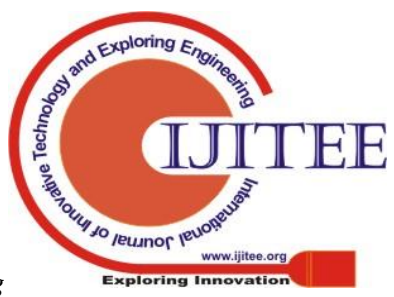


Enhancement in Welding Performance of 2\% Maximum Weld Repair Policy

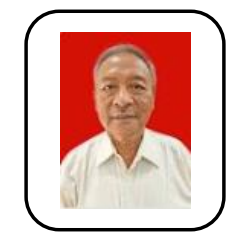

Suparno, as Senior Lecturer at Technology \&

Vocational Eduction Program, Padang State

University, Padang, Indonesia.

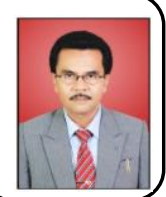

M. Giatman, as Senior Lecturer at Technology \&

Vocational Eduction Program, Padang State University,

Padang, Indonesia 